\title{
Interventions for sexual dysfunction following stroke (Protocol)
}

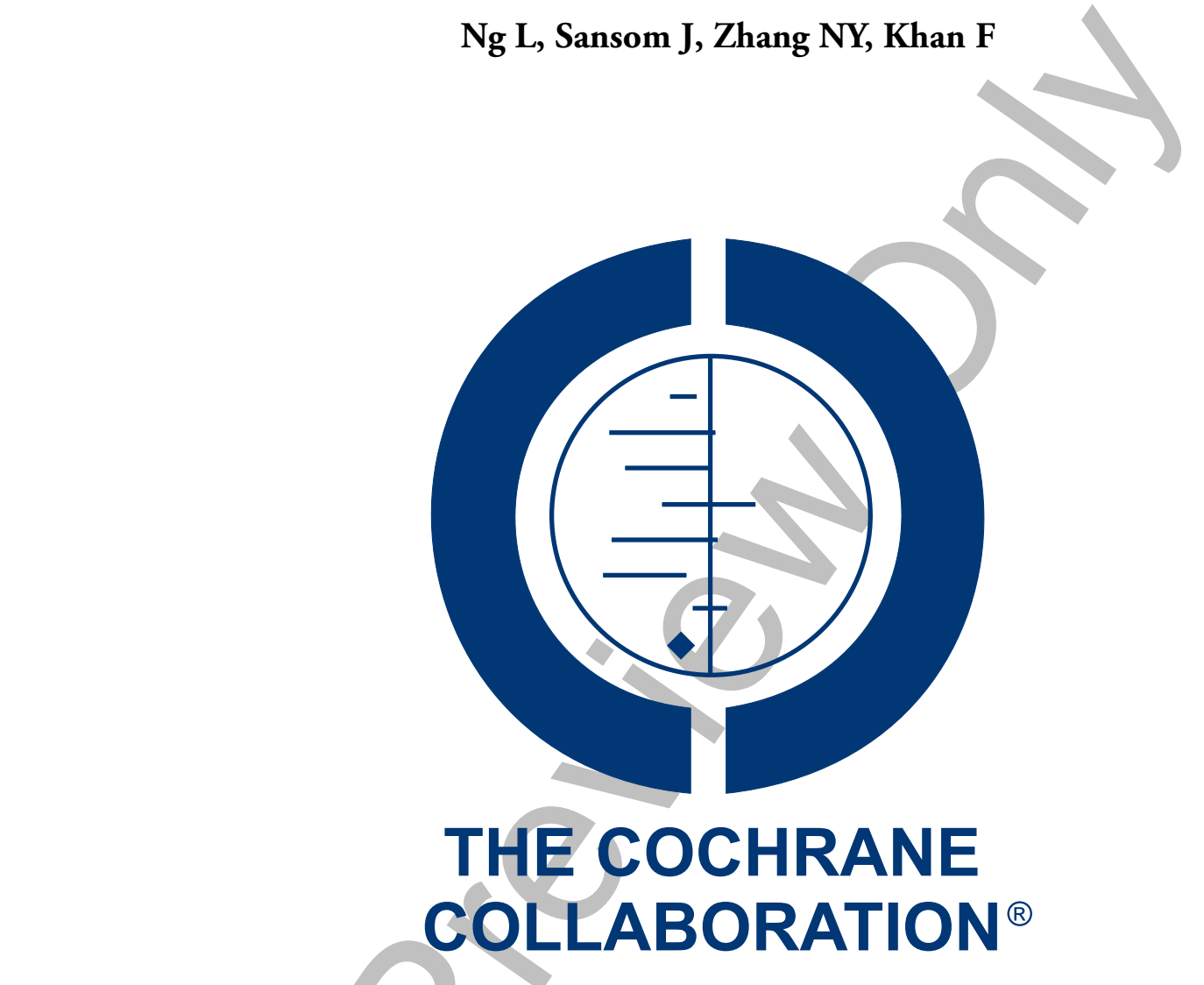

This is a reprint of a Cochrane protocol, prepared and maintained by The Cochrane Collaboration and published in The Cochrane Library 2014, Issue 7

http://www.thecochranelibrary.com

\section{WILEY}


TABLE OF CONTENTS

HEADER . . . . . . . . . . . . . . . . . . . . . . . . . . . . . . . . . . . . 1

ABSTRACT . . . . . . . . . . . . . . . . . . . . . . . . . . . . . . . . . . . . . . . 1

BACKGROUND . . . . . . . . . . . . . . . . . . . . . . . . . . . . . . . . . . . .

OBJECTIVES . . . . . . . . . . . . . . . . . . . . . . . . . . . . . . . . . . . . . . . .

METHODS . . . . . . . . . . . . . . . . . . . . . . . . . . . . . . . . . . . . . . .

ACKNOWLEDGEMENTS . . . . . . . . . . . . . . . . . . . . . . . . . . . . . . . . . . . . . . . .

REFERENCES . . . . . . . . . . . . . . . . . . . . . . . . . . . . . . . . . . . . . 6

APPENDICES . . . . . . . . . . . . . . . . . . . . . . . . . . . . . . . . . . . . . . 8

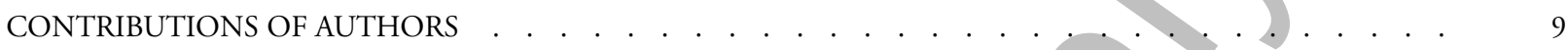

DECLARATIONS OF INTEREST . . . . . . . . . . . . . . . . . . . . . . . . . . . . . . . . . . . . 9

SOURCES OF SUPPORT . . . . . . . . . . . . . . . . . . . . . . . . . . . . . . . . . . . . 10

Interventions for sexual dysfunction following stroke (Protocol)

Copyright $\odot 2014$ The Cochrane Collaboration. Published by John Wiley \& Sons, Ltd. 


\title{
[Intervention Protocol]
}

\section{Interventions for sexual dysfunction following stroke}

\author{
Louisa $\mathrm{Ng}^{1,2}$, Joshua Sansom ${ }^{1,2}$, Nina Y Zhang ${ }^{1}$, Fary Khan ${ }^{1}$ \\ ${ }^{1}$ Department of Rehabilitation Medicine, Royal Melbourne Hospital, Royal Park Campus, Melbourne, Australia. ${ }^{2}$ The University of \\ Melbourne, Parkville, Australia \\ Contact address: Louisa Ng, louisa.ng@mh.org.au.
}

Editorial group: Cochrane Stroke Group.

Publication status and date: New, published in Issue 7, 2014.

Citation: Ng L, Sansom J, Zhang NY, Khan F. Interventions for sexual dysfunction following stroke. Cochrane Database of Systematic Reviews 2014, Issue 7. Art. No.: CD011189. DOI: 10.1002/14651858.CD011189.

Copyright (C) 2014 The Cochrane Collaboration. Published by John Wiley \& Sons, Ltd.

\begin{abstract}
A B S T R A C T
This is the protocol for a review and there is no abstract. The objectives are as follows:

To evaluate the effectiveness of interventions for sexual dysfunction following stroke and to assess adverse events associated with interventions for sexual dysfunction following stroke.
\end{abstract}

\section{B A C K G R O U N D}

Stroke is a leading cause of mortality and disability (ABS 2008). It is estimated that one in six people worldwide will suffer a stroke in their lifetime - in 2012, 50,000 new stroke or recurrent strokes occurred in Australia (NSF 2013), and 795,000 in the USA (CDC 2014). In the same year there were over 420,000 people in Australia and over 7,000,000 people in the USA living with the effects of stroke (NSF 2013; CDC 2014). The burden of disease and economic impact of stroke upon stroke survivors, their caregivers (often family members) and on society is substantial, with lifetime costs per patient ranging from USD 11,787 to USD 3,3035,671 in the USA (Palmer 2005) and annual national costs of AUD 5 billion in Australia (NSF 2013).

\section{Description of the condition}

One of the most common but least talked about effects of stroke is sexual dysfunction with $50 \%$ or more of stroke survivors suffering a degree of sexual decline post-stroke (Korpelainen 1999; Giaquinto 2003; Tamam 2008; Schmitz 2010). Sexual dysfunction is often multifactorial in origin and contributing causes can broadly be divided into the following categories.

- Primary causes where stroke directly affects sexual function, for example, decline in libido and coital frequency for both genders, decline in vaginal lubrication and orgasm in females and in erection and ejaculation in males (Monga 1986; Giaquinto 2003; Tamam 2008).

- Related medical issues, such as medications and premorbid medical conditions (diabetes, hypertension, cardiac issues), may also contribute to these effects (Sjogren 1981; Giaquinto 2003).

- Secondary causes where the stroke results in sensorimotor problems, such as hemiplegia or spasticity, pain, bowel or bladder dysfunction, which in turn affect sexual function due to issues such as the loss of ability to position oneself during sexual activity.

- Tertiary causes such as psychological adjustment issues, or cognitive or behavioural issues or both.

- Psychological adjustment issues may include body image changes, loss of self-esteem, anxiety, stress, depression, fear of new stroke and marital conflict (changes in roles, fear that 
able-bodied partner will leave, difficulties stemming from the spouse having a dual role of lover as well as carer) (Korpelainen 1999; Giaquinto 2003).

- Cognitive or behavioural issues, or both, may include poor judgement, egocentricity, emotional lability, disinhibition, low tolerance for delayed gratification and poor memory.

- Issues may not only relate to the stroke survivor but also to his or her spouse with up to $88 \%$ stating they would not like to have sexual activity with a "sick person" (Giaquinto 2003).

\section{Description of the intervention}

Types of interventions for sexual dysfunction include:

- pharmacological interventions such as phosphodiesterase-5 inhibitors, intracavernosal injections, intraurethral suppositories and hormonal therapy (Vecchio 2010);

- non-pharmacological interventions:

- mechanical devices (such as vacuum pumps, penile implants, penile prostheses and lubricating gels);

- psycho-educational interventions (such as counselling and psychotherapy);

- physical therapy (such as physiotherapy for bed mobility) (Miles 2007);

- complementary medicine interventions such as gingko biloba and ginseng (Miles 2007).

Interventions are not mutually exclusive and may be used in combination. An example of a comprehensive intervention for sexual dysfunction following stroke is sexual rehabilitation. Rehabilitation is defined as "a problem-solving educational process aimed at reducing disability and handicap (participation) experienced by someone as a result of disease or injury" (Wade 1992). The specific aims of stroke sexual rehabilitation are to assess existing sexual issues, provide information on concerns and support safe return to sexual activity after a stroke (Byrne 2014). Sexual rehabilitation is tailored according to individual needs and delivered, in a co-ordinated manner, by medical staff together with one or more disciplines (physiotherapy, occupational therapy, social work, psychologist, nursing). Sexual rehabilitation is designed to be personcentred, time-based, functionally-oriented and aims to maximise activity and participation (social integration) using a biopsychosocial model. Counselling may form a large (and potentially only) component of sexual rehabilitation and may address sexual performance concerns, issues related to medication and co-morbid conditions that may affect sexual function and specific psychological or interpersonal factors (Lue 2004). Counselling may be delivered in a one-on-one or group setting. In addition to counselling, sexual rehabilitation may involve other aspects of physical rehabilitation such as mobility training by the physiotherapist to optimise bed mobility for sexual positioning and transferring in and out of bed, and the management of spasticity such as using a bolster between the knees for adduction spasticity. It may also include the prescrip- tion of medications such as phosphodiesterase- 5 inhibitors. Sexual rehabilitation may be provided by a range of appropriately trained health professionals within the multidisciplinary team and may involve the stroke survivor or his/her partner alone or the stroke survivor together with his or her partner. A range of formats may used in sexual rehabilitation, including oral information, visual information, written materials, audiovisual and practical training. Sexual rehabilitation may be short-term (such as once-off counselling or a medication prescription) or longer-term, for example providing cognitive behavioural therapy targeting psychological and physical aspects of sex and intimacy (Song 2011), or physiotherapy to achieve mobility goals.

At present, a number of international guidelines recommend that assessment and management of sexual function be made following stroke (NSF 2010; RCP 2012; CSN 2014). However, these guidelines are largely based on consensus and do not address types of intervention or their relative effectiveness.

\section{How the intervention might work}

Pharmacological interventions such as phosphodiesterase-5 inhibitors, intracavernosal injections, and intraurethral suppositories assist with erectile function by increasing blood flow to the penis to achieve and maintain erection. Mechanisms of action vary with each medication: phosphodiesterase- 5 inhibitors prevent the breakdown of cyclic guanidine monophosphate (cGMP), which results in enhancement of penile erection, while intracavernosal injections cause vasodilatation of the penis. Hormonal treatment, such as testosterone, treat testosterone deficiency resulting in improved libido and erectile function.

The range of non-pharmacological interventions is broad. Mechanical devices such as vacuum pumps and penile implants/penile prostheses treat erectile dysfunction by using an external pump with a band to obtain and maintain an erection and surgically implanting a prosthesis within the corpora cavernosa of the penis respectively. Lubricating gels reduce friction between body parts, or between body parts and other objects during sexual activity. Psycho-educational interventions (such as counselling and psychotherapy) may reduce anxiety related to sexual problems and provide reassurance around fears related to sexual activity precipitating another stroke resulting in an increase in confidence in sexual abilities. Other therapies may work by providing practical guidance such as ideal timing (sexual activity in the morning when the person is not tired), management of bladder and bowel issues and working around weakness (physical support with pillows) to help stroke survivors and their partners address problems common after a stroke that affect sexuality.

Complementary medicine interventions such as gingko biloba and ginseng may increase nitric oxide levels leading to improved erectile function. 


\section{Why it is important to do this review}

Sexual activity is an integral part of life and the importance of addressing sexual health after stroke is well accepted (NSF 2010). Despite this, a recent Australian National Stroke Audit Rehabilitation Services Report showed that of 2789 post-stroke adults audited across 111 Australian public and private hospitals, only 17\% received information on sexuality (NSF 2012). In addition, although current guidelines recommend the assessment and management of post-stroke sexual dysfunction (NSF 2010), little is known about what type of interventions should be provided and how effective these interventions are. Although some clinical studies and reviews (including Cochrane reviews) have explored the role of these interventions for sexual dysfunction in conditions such as cancer (Miles 2007), chronic kidney disease (Vecchio 2010), and diabetes (Vardi 2007), the effectiveness and safety of these interventions in stroke survivors have not yet been studied thoroughly. This review therefore aims to identify the existing evidence for interventions for sexual dysfunction in stroke survivors, and identify gaps in current knowledge with the purpose of informing health professionals, stroke survivors and their partners, and policy makers about the effectiveness of different interventions.

\section{O B J E C T IVES}

To evaluate the effectiveness of interventions for sexual dysfunction following stroke and to assess adverse events associated with interventions for sexual dysfunction following stroke.

\section{METHODS}

\section{Criteria for considering studies for this review}

\section{Types of studies}

We will include randomised controlled trials (RCTs). We will exclude observational cohort or cross-sectional studies.

\section{Types of participants}

We will include adults aged 18 years and over, male and female, with a definition of stroke as defined by the World Health Organization (WHO) (Hatano 1976). We will also include the partners of the adults following stroke.

We will include studies that include both stroke and non-stroke survivors where at least $75 \%$ of the study population consist of stroke survivors. Where less than $75 \%$ of the study population consist of stroke survivors, we will include these studies if the data relating only to the stroke population is separately reported.

\section{Types of interventions}

We will include trials comparing pharmacological, non-pharmacological or complementary medicine interventions with placebo/ usual care or different forms of intervention (such as a comprehensive individualised psycho-educational programme compared with basic/standard education pamphlet) for treating sexual dysfunction in stroke survivors.

Interventions may include, but are not limited to:

- pharmacological interventions such as phosphodiesterase-5 inhibitors, intracavernosal injections, intraurethral suppositories and hormonal therapy;

- non-pharmacological interventions:

- mechanical devices (such as vacuum pumps, penile implants, penile prostheses and lubricating gels);

- psycho-educational interventions (such as counselling and psychotherapy). This could range from highly structured intervention provided by an appropriately trained health professional (medical practitioner, social worker, occupational therapist, sex therapist) to education alone provided through educational pamphlets from credible sources (such as by established stroke consumer or professional associations as opposed to education from friends who have not undergone appropriate training for such provision of education);

- physical therapy (such as physiotherapy for bed

mobility) by appropriately trained personnel;

- complementary medicine interventions such as gingko biloba and ginseng.

It is anticipated that pharmacological, devices and complementary medicine interventions would generally be compared with placebo. However, other non-pharmacological interventions, such as counselling, could be provided at different intensities or in different formats (face-to-face versus group, written versus verbal). The key characteristic of an intervention is that the intention is to improve sexual dysfunction. We will exclude studies where the intervention might indirectly improve sexual dysfunction but are given primarily for another reason (such as antidepressants for depression).

\section{Types of outcome measures}

\section{Primary outcomes}

The primary outcomes focus on sexual function or sexual satisfaction or both in stroke survivors and their partners.

Instruments that measure sexual function may include:

- International Index of Erectile Function (IIEF) (Rosen 1997);

- Derogatis Interview for Sexual Functioning (DISF) and Derogatis Interview for Sexual Functioning-Self-Report (DISFSR) (Derogatis 1997); 
- Changes in Sexual Functioning Short-Form (CSFQ-14)

(Keller 2006);

- Sexual Function Questionnaire (SFQ) (Quirk 2002);

- Arizona Sexual Experience Scale (ASEX) (McGahuey 2000).

Instruments that measure sexual satisfaction may include:

- Sexual Self-Perception and Adjustment Questionnaire (SSPAQ) (Steinke 2013);

- Sexual Satisfaction Scale for Women (SSS-W) (Meston 2005).

\section{Secondary outcomes}

The secondary outcomes focus on quality of life, psychological well-being (anxiety, depression, stress), satisfaction with sexual rehabilitation services, sexual knowledge and marital/relationship satisfaction in both stroke survivors and their partners.

Instruments that measure quality of life may include:

- 36-item Short Form Health Survey (SF-36) or the 12-item Short Form Health Survey (SF-12) (Ware 1992; Ware 1995);

- Stroke and Aphasia Quality of Life Scale-39 Generic (SAQOL-39g) (Hilari 2003).

Instruments that measure psychological wellbeing may include:

- Depression, Anxiety Stress Scale (DASS) (Lovibond 1995);

- Hospital Anxiety and Depression Scale (HADS) (Zigmond 1983).

Instruments that measure marital/relationship satisfaction include:

- Evaluation and Nurturing Relationship Issues,

Communication and Happiness (ENRICH) Marital Satisfaction (EMS) Scale (Olsen 1993).

We will consider adverse events and side effects that may have resulted from the intervention. We will define serious adverse events as events that were life-threatening (including death) or required prolonged hospitalisation.

\section{Search methods for identification of studies}

See the 'Specialized register' section in the Cochrane Stroke Group module. We will search for trials in all languages and arrange for the translation of relevant articles where necessary.

\section{Electronic searches}

We will search the Cochrane Stroke Group trials register and the following electronic databases:

- Cochrane Central Register of Controlled Trials

(CENTRAL) (The Cochrane Library, latest issue);

- MEDLINE (1950 to present) (Appendix 1);

- EMBASE (1980 to present);
- CINAHL (1982 to present);

- AMED (1985 to present);

- PsycINFO (1806 to present);

- PEDro (Physiotherapy Evidence Database) (http:// www.pedro.org.au/);

- Center for International Rehabilitation Research Information and Exchange (CIRRIE) Database of International Rehabilitation Research (http://cirrie.buffalo.edu/search/ index.php);

- Cochrane Database of Systematic Reviews (The Cochrane Library, latest issue);

- Database of Abstracts of Reviews of Effects (DARE) (The Cochrane Library, latest issue);

- ProQuest Dissertations \& Theses Database (PQDT);

- OT Search by the American Occupational Foundation and the American Occupational Therapy Association (http:// www1.aota.org/otsearch/);

- Occupational Therapy Systematic Evaluation of Evidence (OTseeker) (http://www.otseeker.com/);

- the National Rehabilitation Information Center REHABDATA Database (http://www.naric.com/research/ rehab/);

- SPORTDiscus (http://www.ebscohost.com/public/ sportdiscus);

- ClinicalTrials.gov (http://clinicaltrials.gov/);

- Current Controlled Trials (http://www.controlledtrials.com/);

- Trials Central (http://www.trialscentral.org/);

- The Internet Stroke Center Stroke Trials Registry (http:// www.strokecenter.org/trials);

- UK Clinical Research Network Portfolio database (http:// public.ukcrn.org.uk/search/).

We developed the MEDLINE search strategy with the help of the Cochrane Stroke Group Trials Search Co-ordinator (Appendix 1), and will adapt it for the other databases.

\section{Searching other resources}

To identify further published, unpublished and ongoing trials, we will:

- handsearch the reference lists of included trials and review articles about sexual rehabilitation after stroke;

- track citations using Web of Science Cited Reference Search for all included studies;

- contact experts active in this field (including authors of included trials and excluded studies identified as possible preliminary or pilot work).

\section{Data collection and analysis}




\section{Selection of studies}

Two review authors (LN, JS) will independently screen titles and abstracts of the references obtained as a result of our searching activities and will exclude obviously irrelevant reports. We will retrieve the full-text articles for the remaining references and two review authors (LN, JS) will independently screen the full-text articles and identify studies for inclusion, and identify and record reasons for exclusion of the ineligible studies. We will resolve any disagreements through discussion or, if required, we will consult a third review author (NZ). We will collate multiple reports of the same study so that each study, not each reference, is the unit of interest in the review. We will record the selection process and complete a Preferred Reporting Items for Systematic Reviews and Meta-Analyses (PRISMA) flow diagram.

\section{Data extraction and management}

Two review authors (LN, JS) will independently extract data from included studies. We will group studies by intervention type (pharmacological, non-pharmacological and complementary medicine) where possible.

We will use a pre-designed data extraction form to extract data from the included studies. Two review authors (LN, JS) will independently document the following.

- Participants: number of participants, age, gender, baseline functional status or level of impairment.

- Methods: inclusion criteria, time since stroke, and type, nature and location of lesion.

- Interventions: description of interventions given to each treatment group including the duration, type, dose, route of delivery and frequency. In interventions provided by therapists, we will document the discipline of the person providing the intervention (e.g. physician, occupational therapist, physiotherapist, psychologist, social worker).

- Outcomes: we will document the primary and secondary outcomes relevant to this review. If a study has used different methods of measuring the same outcome, we will note the outcome to be used for any subsequent analysis.

We will note any important confounding variables. If more than two intervention groups are included in the study, we will note the method of including these groups in any subsequent analysis. The two review authors will resolve any data extraction discrepancies through discussion. If disagreement persists, a third author (NZ) will independently extract the data.

\section{Assessment of risk of bias in included studies}

Two review authors (LN, JS) will independently assess risk of bias for each study using the criteria outlined in the Cochrane Handbook for Systematic Reviews of Interventions (Higgins 2011). We will resolve any disagreements by discussion or by involving an- other author (NZ). We will assess the risk of bias according to the following seven domains.

1. Random sequence generation.

2. Allocation concealment.

3. Blinding of participants and personnel.

4. Blinding of outcome assessment.

5. Incomplete outcome data.

6. Selective outcome reporting.

7. Other bias.

We will grade the risk of bias for each domain as high, low or unclear and provide information from the study report together with a justification for our judgment in the 'Risk of bias' tables.

\section{Measures of treatment effect}

We will group studies by intervention type (pharmacological, nonpharmacological and complementary medicine) where possible and analyse them by single or combined interventions to minimise confounding. We will use the Cochrane Review Manager software (Review Manager 2014) to carry out statistical analyses to determine the treatment effect. For dichotomous variables we will calculate the treatment effect using a fixed-effect or randomeffects model and report it as odds ratios (ORs) with 95\% confidence intervals (CIs). For continuous data we will calculate the treatment effect using standardised mean differences (SMDs) and 95\% CIs where different studies used different scales to assess the same outcome, and calculate mean differences (MDs) and 95\% CIs where studies have all used the same method of measuring outcome.

\section{Unit of analysis issues}

The primary outcome of sexual function/sexual satisfaction and secondary outcomes of quality of life, psychological functioning, satisfaction with sexual rehabilitation services, sexual knowledge and marital/relationship satisfaction comprise either ordinal data from measurement scales or continuous data, and we will analyse these as continuous variables. Where reported outcomes have a scale where a lower value indicates a better outcome we will multiply the reported values by -1 so that in all analyses a higher value will indicate a better outcome.

If studies report change values and the baseline value is available, we will calculate the value at follow-up (change value - baseline value). If studies report change values and the baseline value is not available, we will use these data in meta-analyses but plan sensitivity analyses to investigate the effect of including these data. We will analyse adverse events as dichotomous variables.

\section{Dealing with missing data}

If an included study does not report a particular outcome but it has been included in the battery of measures administered, we will contact the authors for the original data. If we are unsuccessful in 
obtaining the data, we will not include that study in the analyses of that outcome.

If an included study has missing data (e.g. reports means but not standard deviations for the follow-up data) we will contact the authors for the missing data. If we are unsuccessful, then we will take logical steps to enter an assumed value. Such steps may include estimating a standard deviation based on a reported standard error, estimating a follow-up standard deviation based on a baseline value, using the median as a proxy for the mean, and using and a multiple of 0.75 times the interquartile range or 0.25 times the range as a proxy for the standard deviation values (Hozo 2005). We plan to do sensitivity analyses to investigate the effect of entering assumed values.

\section{Assessment of heterogeneity}

We will use the $\mathrm{I}^{2}$ statistic to measure heterogeneity among the trials in each analysis. Where there is substantial heterogeneity (as defined by $\mathrm{I}^{2}$ greater than $50 \%$ ), we will perform a meta-analysis using a random-effects model. If $\mathrm{I}^{2}$ is less than or equal to $50 \%$ we will use a fixed-effect meta-analysis.

\section{Assessment of reporting biases}

We will attempt to avoid reporting biases by using a comprehensive search strategy that includes searching for unpublished studies and searching trials registers. We will also assess the completeness of outcome data.

\section{Data synthesis}

Where we consider studies to be sufficiently similar, we will conduct a meta-analysis by pooling the appropriate data using RevMan 5.2 (Review Manager 2014).

\section{Subgroup analysis and investigation of heterogeneity}

We intend to explore heterogeneity by additional subgroup analyses to investigate the effect of:

- time since stroke;

- type of intervention;

- level of impairment at baseline;

- adherence with additional intervention.

\section{Sensitivity analysis}

We intend to carry out a sensitivity analysis (if necessary) to explore the effect of the following methodological features.

- Allocation concealment: we will re-analyse data, excluding trials with inadequate or unclear allocation concealment.

- Masking of outcome assessor: we will re-analyse data, excluding trials without or with unclear masking of outcome assessor.

- Missing outcome data: we will re-analyse the data, excluding trials with inadequate or unclear methods of dealing with missing outcome data.

\section{ACKNOWLEDGEMENTS}

We would like to acknowledge the Cochrane Stroke Group for their assistance.

\section{R E F E R E N C E S}

\section{Additional references}

ABS 2008

Australian Bureau of Statistics. 3303.0 - Causes of Death, Australia, 2006. Released 19 March 2008. http: //www.abs.gov.au/AUSSTATS/abs@.nsf/DetailsPage/ 3303.02006? OpenDocument.

Byrne 2014

Byrne M, Doherty S, Fridlund BGA, Martensson J, Steinke EE, Jaarsma T, et al.Sexual counselling for sexual problems in patients with cardiovascular disease. Cochrane Database of Systematic Reviews 2014, Issue 2. [DOI: 10.1002/ 14651858.CD010988]

CDC 2014

Centers for Disease Control and Prevention. National Center for Chronic Disease Prevention and Health Promotion, Division for Heart Disease and Stroke
Prevention (updated March 17, 2014). Stroke Facts. http:// www.cdc.gov/stroke/facts.htm.

CSN 2014

Canadian Stroke Network and the Heart and Stroke Foundation of Canada. Canadian Best Practice Recommendations for Stroke Care. http:// www.strokebestpractices.ca/ 2014.

Derogatis 1997

Derogatis LR. The Derogatis Interview for Sexual Functioning (DISF/DISF-SR): an introductory report. Journal of Sex and Marital Therapy 1997;23(4):291-304.

Giaquinto 2003 Giaquinto S, Buzzelli S, Di Francesco L, Nolfe G. Evaluation of sexual changes after stroke. Journal of Clinical Psychiatry 2003;64(3):302-7.

Hatano 1976

Hatano S. Experience from a multicentre stroke register: 
a preliminary report. Bulletin of the World Health

Organization 1976;54(5):541-53.

\section{Higgins 2011}

Higgins JPT, Green S (editors). Cochrane Handbook for Systematic Reviews of Interventions Version 5.1.0 [updated March 2011]. The Cochrane Collaboration, 2011. Available from www.cochrane-handbook.org.

\section{Hilari 2003}

Hilari K, Byng S, Lamping DL, Smith SC. Stroke and Aphasia Quality of Life Scale-39 (SAQOL-39): evaluation of acceptability, reliability, and validity. Stroke 2003;34(8): 1944-50.

\section{Hozo 2005}

Hozo SP, Djulbegovic B, Hozo I. Estimating the mean and variance from the median, range, and the size of a sample. BMC Medical Research Methodology 2005;1(5):13.

\section{Keller 2006}

Keller A, McGarvey EL, Clayton AH. Reliability and construct validity of the Changes in Sexual Functioning Questionnaire short-form (CSFQ-14). Journal of Sex and Marital Therapy 2006;32(1):43-52.

\section{Korpelainen 1999}

Korpelainen JT, Nieminen P, Myllyla VV. Sexual functioning among stroke patients and their spouses. Stroke 1999;30(4): 715-9.

\section{Lovibond 1995}

Lovibond SH, Lovibond PF. Manual for the Depression Anxiety Stress Scales. 2nd Edition. Sydney: Psychology Foundation, 1995.

Lue 2004

Lue TF, Giuliano F, Montorsi F, Rosen RC, Andersson KE, Althof S. Summary of the recommendations on sexual dysfunctions in men. Journal of Sexual Medicine 2004;1: 6-23.

McGahuey 2000

McGahuey CA, Gelenberg AJ, Laukes CA, Moreno FA, Delgado PL, McKnight KM, et al.The Arizona Sexual Experience Scale (ASEX): reliability and validity. Journal of Sex and Marital Therapy 2000;26(1):25-40.

\section{Meston 2005}

Meston C, Trapnell P. Development and validation of a five-factor sexual satisfaction and distress scale for women: the Sexual Satisfaction Scale for Women (SSS-W). Journal of Sexual Medicine 2005;2(1):66-81.

\section{Miles 2007}

Miles CL, Candy B, Jones L, Williams R, Tookman A, King M. Interventions for sexual dysfunction following treatments for cancer. Cochrane Database of Systematic Reviews 2007, Issue 4. [DOI: 10.1002/14651858.CD005540.pub2]

\section{Monga 1986}

Monga TN, Lawson JS, Inglis J. Sexual dysfunction in stroke patients. Archives of Physical Medicine and Rehabilitation 1986;67(1):19-22.

\section{NSF 2010}

National Stroke Foundation. Clinical Guidelines for Stroke Management 2010. Melbourne, Australia. Available from http://strokefoundation.com.au/health-professionals/toolsand-resources/clinical-guidelines-for-stroke-preventionand-management/.

\section{NSF 2012}

National Stroke Foundation. National Stroke AuditRehabilitation Services Report 2012. Melbourne, Australia. Available from http://strokefoundation.com.au/healthprofessionals/tools-and-resources/national-stroke-auditreports/.

\section{NSF 2013}

National Stroke Foundation. Deloitte Access Economics Report. The economic impact of stroke in Australia. 2013. Melbourne, Australia. Available from http:// strokefoundation.com.au/2013/03/the-economic-impactof-stroke-in-australia/.

\section{Olsen 1993}

Olsen D, Fowers BJ. Enrich Marital Satisfaction Scale: a brief research and clinical tool. Journal of Family Psychology 1993;7:176-85.

\section{Palmer 2005}

Palmer AJ, Valentine WJ. Overview of costs of stroke. Current Medical Research and Opinion 2005;21:19-26.

\section{Quirk 2002}

Quirk FH, Heiman JR, Rosen RC, Laan E, Smith MD, Boolell M. Development of a sexual function questionnaire for clinical trials of female sexual dysfunction. Journal of Womens Health and Gender Based Medicine 2002;11(3): 277-89.

\section{RCP 2012}

Intercollegiate Stroke Working Party. National clinical guideline for stroke, 4th edition. London: Royal College of Physicians, 2012. Available from http:// www.rcplondon.ac.uk/resources/stroke-guidelines. London.

\section{Review Manager 2014}

The Nordic Cochrane Centre, The Cochrane Collaboration. Review Manager (RevMan). 5.3. Copenhagen: The Nordic Cochrane Centre, The Cochrane Collaboration, 2014.

\section{Rosen 1997}

Rosen RC, Riley A, Wagner G, Osterloh IH, Kirkpatrick J, Mishra A. The international index of erectile function (IIEF): a multidimensional scale for assessment of erectile dysfunction. Urology 1997;49(6):822-30.

\section{Schmitz 2010}

Schmitz MA, Finkelstein M. Perspectives on poststroke sexual issues and rehabilitation needs. Topics in Stroke Rehabilitation 2010;17(3):204-13.

\section{Sjogren 1981}

Sjogren K, Fugl-Meyer AR. Sexual problems in hemiplegia. Internal Journal of Rehabilitation Medicine 1981;3(1):26-31. 
Song 2011

Song H, Oh H, Kim H, Seo W. Effects of a sexual rehabilitation intervention program on stroke patients and their spouses. NeuroRehabilitation 2011;28(2):143-50.

Steinke 2013

Steinke EE, Mosack V, Hill TJ. Sexual self-perception and adjustment of cardiac patients: a psychometric analysis. Journal of Research in Nursing 2013;18(3):191-201.

Tamam 2008

Tamam Y, Tamam L, Akil E, Yasan A, Tamam B. Poststroke sexual functioning in first stroke patients. European Journal of Neurology 2008;15(7):660-6.

Vardi 2007

Vardi M, Nini A. Phosphodiesterase inhibitors for erectile dysfunction in patients with diabetes mellitus. Cochrane Database of Systematic Reviews 2007, Issue 1. [DOI: 10.1002/14651858.CD002187.pub3]

Vecchio 2010

Vecchio M, Navaneethan SD, Johnson DW, Lucisano G, Graziano G, Saglimbene V, et al.Interventions for treating sexual dysfunction in patients with chronic kidney disease. Cochrane Database of Systematic Reviews 2010, Issue 12. [DOI: 10.1002/14651858.CD007747.pub2]

Wade 1992

Wade DT. Measurement in Neurology Rehabilitation. Oxford: Oxford University Press, 1992.

\section{Ware 1992}

Ware JE Jr, Sherbourne CD. The MOS 36-item short-form health survey (SF-36). I. Conceptual framework and item selection. Medical Care 1992;30(6):473-83.

\section{Ware 1995}

Ware JE Jr, Kosinski M, Bayliss MS, McHorney CA, Rogers WH, Raczek A. Comparison of methods for the scoring and statistical analysis of SF-36 health profile and summary measures: summary of results from the Medical Outcomes Study. Medical Care 1995;33(4 Suppl):AS264-79.

Zigmond 1983

Zigmond AS, Snaith RP. The hospital anxiety and depression scale. Acta Psychiatrica Scandinavica 1983;67(6): $361-70$.

* Indicates the major publication for the study

\section{A P P E N D I C E S}

\section{Appendix I. MEDLINE search strategy}

1. cerebrovascular disorders/ or exp basal ganglia cerebrovascular disease/ or exp brain ischemia/ or exp carotid artery diseases/ or exp cerebrovascular trauma/ or exp intracranial arterial diseases/ or exp intracranial arteriovenous malformations/ or exp "intracranial embolism and thrombosis"/ or exp intracranial hemorrhages/ or stroke/ or exp brain infarction/ or stroke, lacunar/ or vasospasm, intracranial/ or vertebral artery dissection/

2. (stroke $\$$ or post stroke or poststroke or post-stroke or apoplex\$ or cerebral vasc $\$$ or cerebrovasc $\$$ or cva or SAH).tw.

3. ( (brain or cerebr $\$$ or cerebell\$ or vertebrobasil $\$$ or hemispher $\$$ or intracran $\$$ or intracerebral or infratentorial or supratentorial or middle cerebr $\$$ or mca $\$$ or anterior circulation or basilar artery or vertebral artery) adj5 (isch?emi $\$$ or infarct $\$$ or thrombo $\$$ or emboli $\$$ or occlus\$ or hypoxi\$)).tw.

4. ((brain $\$$ or cerebr $\$$ or cerebell $\$$ or intracerebral or intracran $\$$ or parenchymal or intraparenchymal or intraventricular or infratentorial or supratentorial or basal gangli $\$$ or putaminal or putamen or posterior fossa or hemispher\$ or subarachnoid) adj5 (h?emorrhag $\$$ or h? ematoma $\$$ or bleed $\$)$ ).tw.

5. exp hemiplegia/ or exp paresis/ or gait disorders, neurologic/

6. exp aphasia/ or hemianopsia/

7. (hempar\$ or hemipleg\$ or paresis or paretic or aphas* or dysphas\$ or hemianop\$).tw.

8. or/ $1-7$

9. exp sexual behavior/ or sex counseling/ or libido/ or exp sexual dysfunction, physiological/ or exp sexual dysfunctions, psychological/ or sexual partners/

10. ((sexual\$ or libido) adj5 (disorder\$ or declin\$ or dysfunct\$ or impair\$ or deficit\$ or disabilit\$ or problem\$ or concern\$ or difficult\$)).tw.

11. (sexual\$ adj5 (activ\$ or health or issue $\$$ or satisfaction or function\$ or experience $\$$ or adjustment or chang $\$$ or knowledge or relation $\$$ or skill $\$$ or intercourse or interest $\$$ or wish $\$$ or need $\$$ or behav $\$$ or perform $\$$ or spouse or partner or wife or husband)).tw. 12. (sexual $\$$ adj5 (therap $\$$ or treat $\$$ or rehab $\$$ or train $\$$ or counsel $\$$ or psycholog $\$$ or psychother $\$$ or physiotherapy $\$$ or help $\$$ or advi $\$$ or inform $\$$ or guid $\$$ or intervention $\$$ or educat $\$)$ ).tw. 
13. (erectile adj3 (function\$ or dysfunction\$)).tw.

14. (intima $\$$ adj3 (relation $\$$ or spouse or partner or wife or husband)).tw.

15. (marital adj3 relation\$).tw.

16. 9 or 10 or 11 or 12 or 13 or 14 or 15

17. Randomized Controlled Trials as Topic/

18. random allocation/

19. Controlled Clinical Trials as Topic/

20. control groups/

21. clinical trials as topic/

22. double-blind method/

23. single-blind method/

24. Placebos/

25. placebo effect/

26. cross-over studies/

27. Drug Evaluation/ or drug therapy.fs.

28. therapies, investigational/ or research design/

29. randomized controlled trial.pt.

30. controlled clinical trial.pt.

31. clinical trial.pt.

32. (random $\$$ or RCT or RCTs).tw.

33. (controlled adj5 (trial\$ or stud\$)).tw.

34. (clinical\$ adj5 trial\$).tw.

35. ((control or treatment or experiment\$ or intervention) adj5 (group\$ or subject\$ or patient\$)).tw.

36. (quasi-random $\$$ or quasi random $\$$ or pseudo-random $\$$ or pseudo random $\$$ ).tw.

37. ((control or experiment $\$$ or conservative) adj5 (treatment or therapy or procedure or manage $\$)$ ).tw.

38. ((singl\$ or doubl\$ or tripl\$ or trebl\$) adj5 (blind\$ or mask\$)).tw.

39. (cross-over or cross over or crossover).tw.

40. (placebo\$ or sham).tw.

41. trial.tw

42. (assign\$ or allocat\$).tw.

43. (controls or groups).ab.

44. or/17-43

45. 8 and 16 and 44

46. exp animals/ not humans.sh.

47. 45 not 46

\section{CONTRIBUTIONS OFAUTHORS}

Louisa $\mathrm{Ng}$ will lead this review, run the searches, identify relevant articles, assist with data extraction, review the papers, provide methodological and content expertise and write the drafts of the review.

Joshua Sansom will identify relevant articles, assist with data extraction, review the papers and write the drafts of the review as a second review author.

Nina Zhang will provide content expertise and arbitrate where necessary during the identification of relevant articles, data extraction and review of papers.

Fary Khan will provide content and methodological expertise as well as comments on the final drafts of the review.

Interventions for sexual dysfunction following stroke (Protocol)

Copyright @ 2014 The Cochrane Collaboration. Published by John Wiley \& Sons, Ltd. 


\section{DECLARATIONSOF INTEREST}

None known.

\section{SOURCES OF SUPPORT}

\section{Internal sources}

- Royal Melbourne Hospital, Australia.

\section{External sources}

- No sources of support supplied 


\section{University Library}

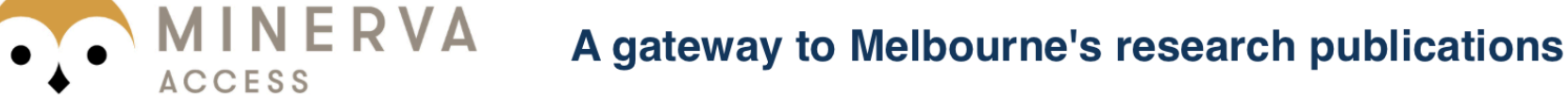

Minerva Access is the Institutional Repository of The University of Melbourne

Author/s:

NG, L;Sansom, J;Zhang, N;Khan, F

Title:

Interventions for sexual dysfunction following stroke (Intervention Protocol)

Date:

2014

Citation:

NG, L., Sansom, J., Zhang, N. \& Khan, F. (2014). Interventions for sexual dysfunction

following stroke (Intervention Protocol). Cochrane Database of Systematic Reviews, 2014 (7), https://doi.org/10.1002/14651858.CD011189.

Persistent Link:

http://hdl.handle.net/11343/118541 